\title{
Synthesis of Co-oligomers of Thiophene Derivatives from Oligoynes via Zirconacyclopentadiene
}

\author{
Debabrata Payra, ${ }^{1,2}$ Zhiyi Song, ${ }^{1}$ Ken-ichiro Kanno, ${ }^{1}$ and Tamotsu Takahashi*1 \\ ${ }^{1}$ Catalysis Research Center, Hokkaido University, Kita 21, Nishi 10, Kita-ku, Sapporo, Hokkaido 001-0021 \\ ${ }^{2}$ Asian Graduate Schools of Chemistry and Materials Science, Hokkaido University, \\ Kita 13, Nishi 8, Kita-ku, Sapporo, Hokkaido 060-8628
}

(Received August 3, 2011; CL-110651; E-mail: tamotsu@cat.hokudai.ac.jp)

For the formation of oligothiophenes or thiophene cooligomers, a preconnected method using zirconacyclopentadienes was demonstrated. Oligoynes of 4,4-bis(butoxymethyl)-1,6-heptadiyne linked through bithiophene were prepared where all components and diyne moieties were connected in advance before converting to zirconacyclopentadiene-containing precursors. Treatment of the reactive zirconacyclopentadienes in the preconnected precursors with acid, dimethyl acetylenedicarboxylate or sulfur monochloride afforded different oligothiophenes or thiophene co-oligomer derivatives.

Oligomers of thiophene or co-oligomers of thiophene with other aromatic components have attracted much attention in relevance to electronic organic materials. ${ }^{1}$ Synthetic methodology of these oligomers or co-oligomers is an interesting topic. The most general conventional method is the connection of separate components in the presence of a catalytic or equivalent amount of metal compounds as shown in Scheme $1 .^{2}$ Previously our group and others have demonstrated a $\mathrm{Zr}$-mediated method which involves the conversion of the zirconacyclopentadiene moiety in the precursors into some component as shown in Scheme 1. ${ }^{3}$

We have reported Ni-catalyzed polymerization of dibromoterphenyl units. ${ }^{3 a}$ The dibromoterphenyl unit was prepared by this zirconium-mediated method. For the formation of the phenylene copolymers, Tilley and co-workers have reported a method via zirconium-containing phenylene precursors which were prepared by coupling of phenyl-bridged diyne units on zirconium (Scheme 2). ${ }^{3 b}$ Herein, we would like to report a novel method for preparation of zirconium-containing precursors using a preconnected method (Scheme 2) for constructing oligomers of thiophene or co-oligomers of thiophene and other conjugated fragments. The preconnected method helped to produce a welldefined conjugated backbone with complete regioselectivity of zirconacyclopentadienes. This is in sharp contrast to a coupling method on zirconium which gives a mixture of several components and regioisomers.

Preconnected oligodiynes were prepared by the SonogashiraHagihara coupling of a terminal diyne and bromo bithiophene. 2,2'-Bithiophene was chosen as an oligothiophene and 4,4bis(butoxymethyl)-1,6-heptadiyne as a terminal diyne unit. By careful control of the equivalent ratio of bromo substrate and

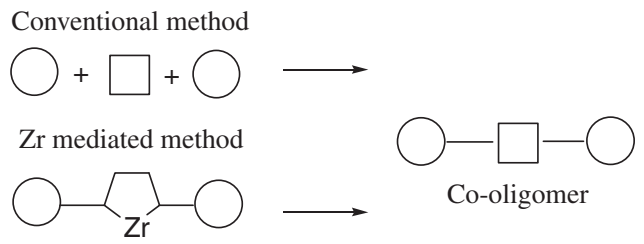

Scheme 1. Co-oligomer preparation. diyne, oligodiyne 1a, $\mathbf{1 b}$, and 1c were prepared in $83 \%, 85 \%$, and $64 \%$ yields, respectively (Scheme 3 ). ${ }^{4}$ These oligodiynes were isolated as viscous light to dark yellow oils. All oligodiynes were well soluble in common organic solvents such as THF, $\mathrm{CHCl}_{3}$, or EtOAc and fully characterized by ${ }^{1} \mathrm{HNMR},{ }^{13} \mathrm{CNMR}$, and HRMS.

Oligodiynes 1a, 1b, or 1c were treated with one, two, or three equiv of $\mathrm{Cp}_{2} \mathrm{ZrBu}_{2}$ (Negishi reagent) ${ }^{5}$ which was prepared in situ by the reaction of $\mathrm{Cp}_{2} \mathrm{ZrCl}_{2}$ with 2 equiv of $n-\mathrm{BuLi}$ in THF at

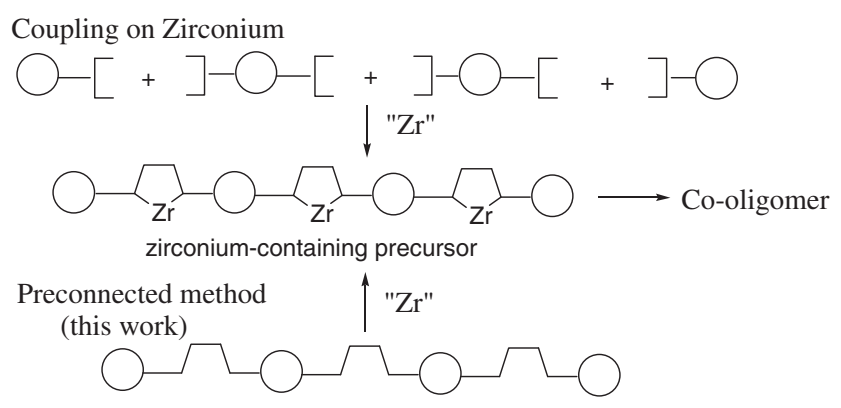

Scheme 2. Preparation methods for zirconium-containing precursors.

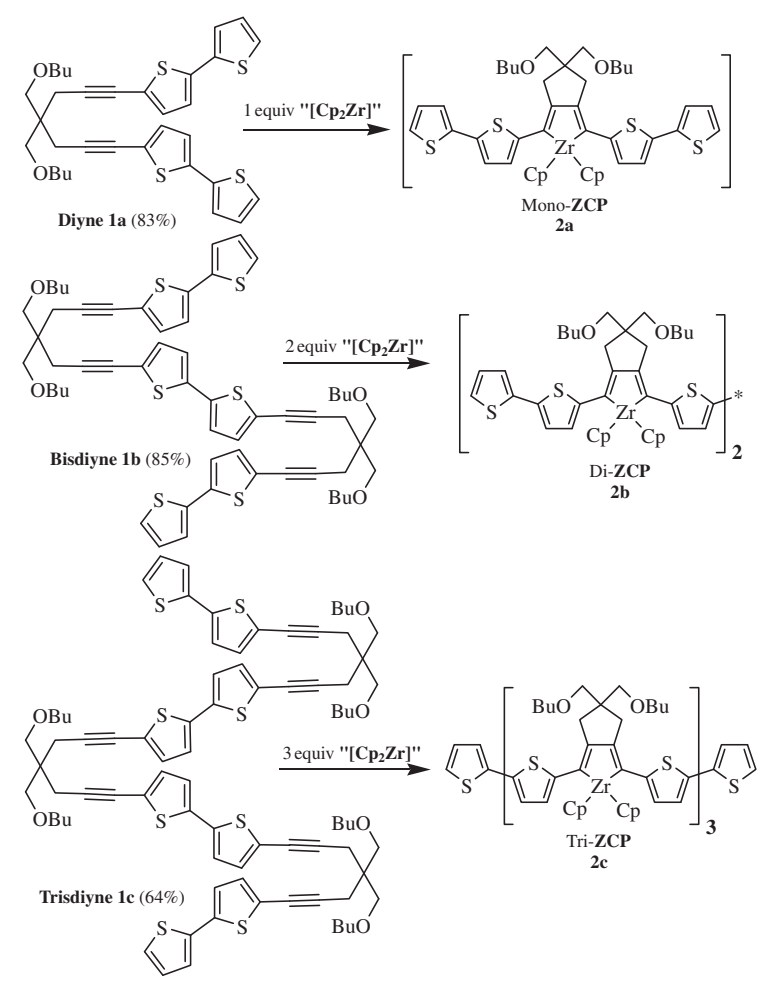

Scheme 3. Oligomers of diyne and ZCP connected by thiophenes. 


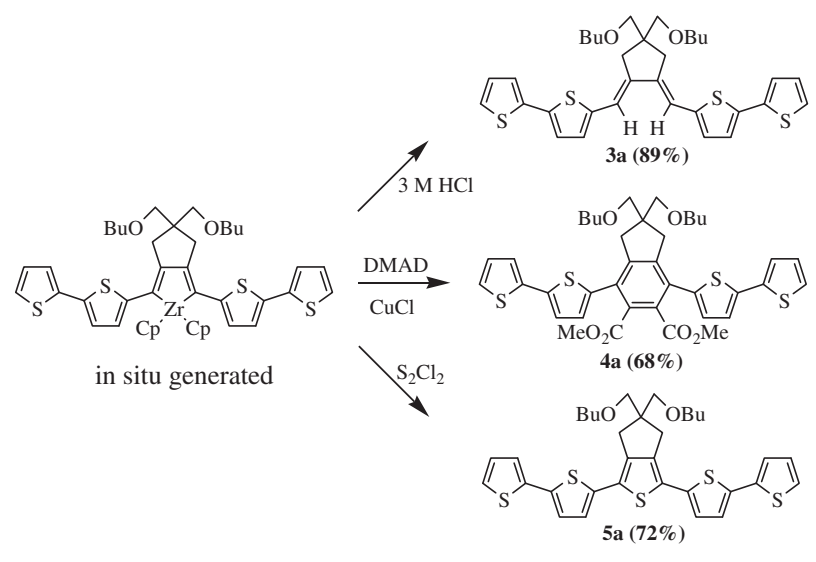

Scheme 4. Reactions of active ZCP-containing thiophenes.

$-78^{\circ} \mathrm{C}$ for $1 \mathrm{~h}$, and stirred at room temperature for $5 \mathrm{~h}$ to afford the corresponding zirconacyclopentadiene (ZCP)-inserted oligothiophene intermediates $\mathbf{2 a}, \mathbf{2 b}$, or $\mathbf{2 c}$ almost quantitatively (monitored by TLC). Noticeably, cyclization occurred exclusively intramolecularly among adjacent diynes. The presence of active zirconacycle intermediate was confirmed by ${ }^{1} \mathrm{HNMR}$ and ${ }^{13} \mathrm{CNMR}$ spectra. The colors of the ZCP intermediates were observed to be greatly changed depending on the conjugation length, for instance, monomer was deep yellow but dimer and trimer were red and violet color respectively.

Intermediate ZCP is very reactive toward a large number of substrates like alkynes, nitriles, or halides of a variety of main group elements (germanium, tin, phosphorus, sulfur, etc.) to construct aromatic or heteroaromatic fragment in situ. ${ }^{6}$ So far, we succeeded in converting ZCP-containing oligomers to three kinds of oligothiophene derivatives (Scheme 4) under the given set of conditions. Protonation of ZCP intermediate $2 \mathrm{a}$ with $3 \mathrm{M}$ aqueous $\mathrm{HCl}$ produced oligothiophene derivative 3a as a deep yellow powder in $89 \%$ yield. In the backbone of oligomer $\mathbf{3 a}$, there is a 1,3-butadiene unit bridging the thiophene fragments. Such butadiene unit is more flexible than the aromatic rings and could be further manipulated to various derivatives by deprotonation or cycloaddition reactions. Previously we reported that coupling of a zirconacycle with a third alkyne like dimethyl acetylenedicarboxylate (DMAD) in the presence of $\mathrm{CuCl}$ gives benzene derivative. ${ }^{7}$ A similar procedure worked in $\mathbf{2 a}$ to afford phenylene-thiophene co-oligomer $4 \mathbf{a}$ in $68 \%$ isolated yield. By employing different kinds of third alkyne we could produce a large variety of phenylene derivatives, furthermore, the functional groups on the phenylene unit could be utilized for further structural modification. When mono-ZCP 2a was treated with sulfur monochloride $\left(\mathrm{S}_{2} \mathrm{Cl}_{2}\right)^{8}$ at room temperature, zirconacycle was converted to a new thiophene ring which lead to an all-thiophene chain. Pentathiophene derivative 5a was isolated as an orange-yellow powder in $72 \%$ yield. These results indicated that our thiopheneconjugated ZCP is very reactive toward different kind of substrates. Further, we envision that this zirconacycle could be easily replaced by other heterocycles like phosphole, silole, germole, and so on. ${ }^{8}$

Being encouraged by the reactivity of mono-ZCP $\mathbf{2 a}$, we became interested in exploring similar reactions of di-ZCP $\mathbf{2 b}$ and tri-ZCP 2c (Figure 1). Protonation of di- and tri-ZCP produced multiple butadiene unit connected oligothiophene derivatives $\mathbf{3 b}$ and $3 \mathbf{c}$ respectively. These products were isolated as red or violet

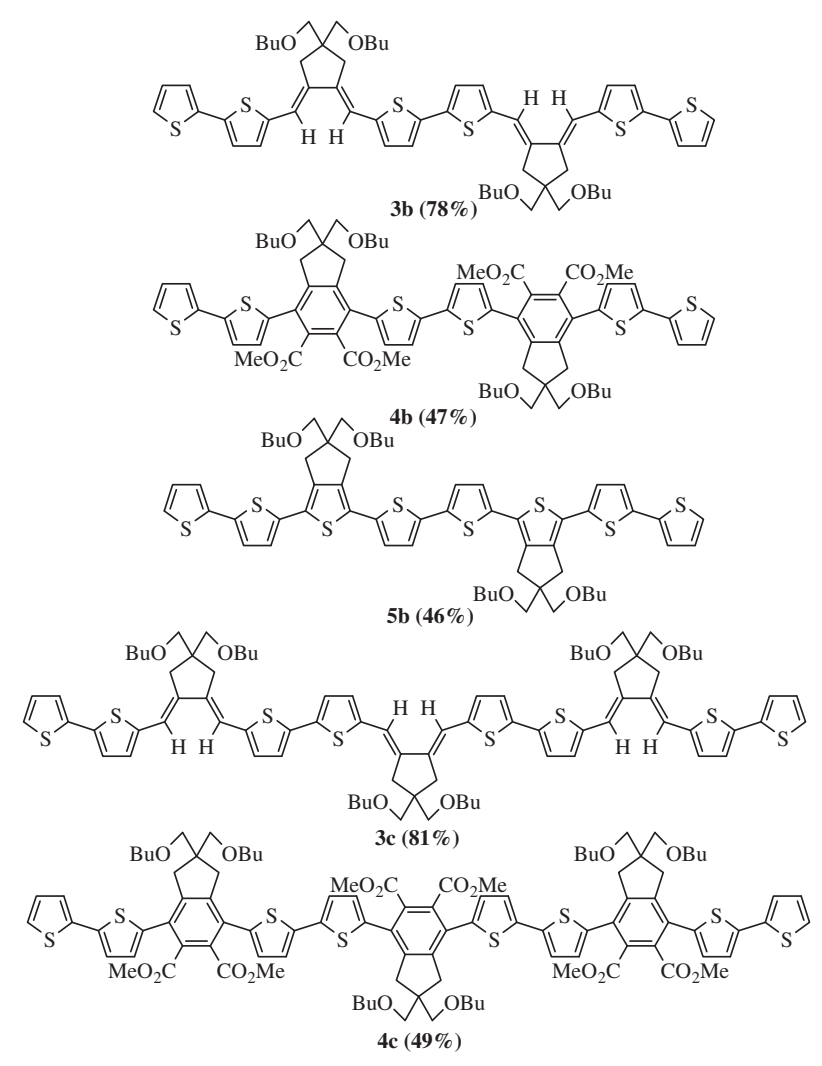

Figure 1. Co-oligomers 3, 4, and 5 .

colored powder in $78 \%$ and $81 \%$ yield. Cycloaddition reaction with DMAD also proceeded smoothly to afford phenylenethiophene co-oligomers $\mathbf{4 b}$ and $\mathbf{4 c}$ in $47 \%$ and $49 \%$ isolated yields, respectively. Furthermore, the reaction of $\mathrm{S}_{2} \mathrm{Cl}_{2}$ with diZCP formed two new thiophene rings in situ which lead to an octathiophene derivative $\mathbf{5 b}$. This derivative was isolated as a deep red powder in $46 \%$ yield. All the derivatives were air stable. They were well soluble in common organic solvents like $\mathrm{CHCl}_{3}$ or EtOAc due to the presence of $O$-butylated groups. All the products were purified and fully characterized by ${ }^{1} \mathrm{HNMR}$, ${ }^{13} \mathrm{C} \mathrm{NMR}$, and MS.

The optical behavior of the prepared oligothiophene derivatives were investigated by measurements of UV-vis absorption and emission spectra in chloroform (Table 1). The absorption wavelength $\left(\lambda_{\text {abs }}\right)$ and molar extinction coefficient $(\varepsilon)$ of oligothiophene derivatives were observed to increase with increasing effective conjugation length which is consistent with the normal trend. Protonated oligothiophene derivatives $\mathbf{3 a}, \mathbf{3 b}$, and $3 \mathbf{c}$ have an intense absorption band at 441, 497, and $515 \mathrm{~nm}$ respectively. The absorption maximum was red-shifted by $56 \mathrm{~nm}$ for dimer $\mathbf{3 b}$ to monomer $\mathbf{3 a}$ but $18 \mathrm{~nm}$ for trimer $\mathbf{3 c}$ to dimer $\mathbf{3 b}$ (Figure 2). The molar extinction coefficient was found to increase greatly with increasing conjugation length. They do not have significant emission in chloroform. Benzene derivatives $4 \mathbf{a}, \mathbf{4 b}$, and $\mathbf{4 c}$ absorb at 341,354 , and $358 \mathrm{~nm}$ respectively and all emit at $473 \mathrm{~nm}$ (Figure 3). Absorption and emission wavelengths of benzene derivatives were not much different between monomer and oligomers but extinction coefficient and quantum yield ${ }^{9}$ were increasing with longer conjugation length. Penta- and octathiophene derivatives $\mathbf{5 a}$ and $\mathbf{5 b}$ have intense absorption maxima at 
Table 1. Optical properties of oligo-, co-oligothiophene derivatives ${ }^{\mathrm{a}}$

\begin{tabular}{|c|c|c|c|c|c|}
\hline \multirow[b]{2}{*}{ Compound } & & \multicolumn{2}{|c|}{ UV-vis } & \multicolumn{2}{|c|}{ Emission } \\
\hline & & $\begin{array}{l}\lambda_{\max } \\
/ \mathrm{nm}\end{array}$ & $\begin{array}{c}\varepsilon \\
/ \mathrm{M}^{-1} \mathrm{~cm}^{-1 \mathrm{~b}}\end{array}$ & $\begin{array}{l}\lambda_{\mathrm{em}} \\
/ \mathrm{nm}\end{array}$ & $\Phi_{\mathrm{F}}$ \\
\hline \multirow{3}{*}{$\begin{array}{l}\text { Protonated } \\
\text { derivatives }^{\mathrm{c}}\end{array}$} & $3 a$ & 441 & 33660 & - & - \\
\hline & $3 b$ & 497 & 118900 & - & - \\
\hline & $3 c$ & 515 & 144700 & - & - \\
\hline \multirow{3}{*}{$\begin{array}{l}\text { Benzene } \\
\text { derivatives }^{\mathrm{d}}\end{array}$} & $4 a$ & 341 & 32700 & 473 & 0.16 \\
\hline & $4 b$ & 354 & 72520 & 473 & 0.20 \\
\hline & $4 \mathrm{c}$ & 358 & 159200 & 473 & 0.23 \\
\hline \multirow{2}{*}{$\begin{array}{l}\text { All-thiophene } \\
\text { derivatives }\end{array}$} & $5 a$ & 429 & 37370 & 486 & 0.20 \\
\hline & $5 b$ & 475 & 70670 & 543 & 0.16 \\
\hline
\end{tabular}

${ }^{\mathrm{a}} \mathrm{All}$ spectra were measured in $\mathrm{CHCl}_{3}$ at $\mathrm{rt}$. ${ }^{\mathrm{b}}$ Molar extinction coefficient. 'Protonated derivatives do not have significant emission. ${ }^{\mathrm{d}}$ Quantum yield was estimated by comparison with standard anthracene in cyclohexane $\left(\Phi_{\mathrm{F}}=0.36\right)$. ${ }^{\mathrm{e}} \mathrm{Quantum}$ yield was estimated by comparison with standard fluorescien in ethanol $\left(\Phi_{\mathrm{F}}=0.79\right)$.

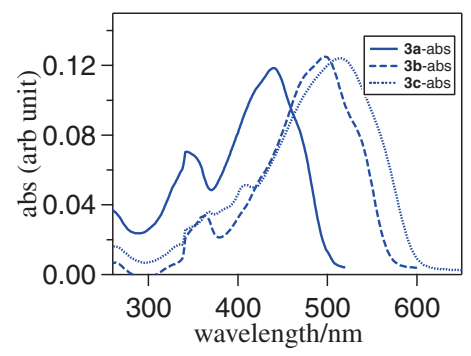

Figure 2. Comparison of absorption spectra among protonated derivatives in $\mathrm{CHCl}_{3}$ at rt.

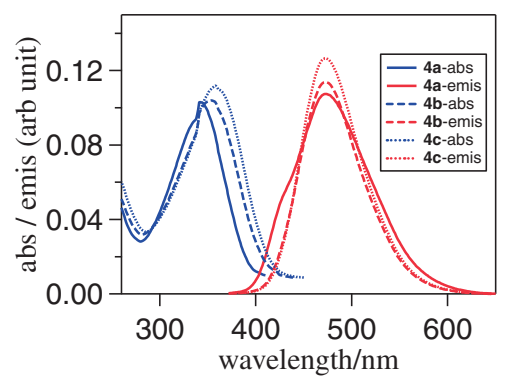

Figure 3. Comparison of absorption and emission spectra of phenylene-thiophene co-oligomers in $\mathrm{CHCl}_{3}$ at rt.

429 and $475 \mathrm{~nm}$. That indicates $\lambda_{\mathrm{abs}}$ for dimer was red-shifted by $46 \mathrm{~nm}$ than monomer. These all-thiophene derivatives $\mathbf{5 a}$ and $\mathbf{5 b}$ intensely emit at 486 and $543 \mathrm{~nm}$ respectively (Figure 4). Unlike the benzene derivatives, $\lambda_{\mathrm{abs}}$ and $\lambda_{\mathrm{em}}$ for all-thiophene derivatives were observed to change greatly for increasing conjugation length besides they have significantly higher absorption and emission wavelength which indicates shorter optical energy gap than phenylene-thiophene co-oligomers. Further studies of these oligothiophene derivatives are in progress.

In summary, we have demonstrated a preconnected approach to access a wide range of thiophene co-oligomers. This method always produces precise conjugated systems which helps to get very pure product and permits systematic study of the structure-

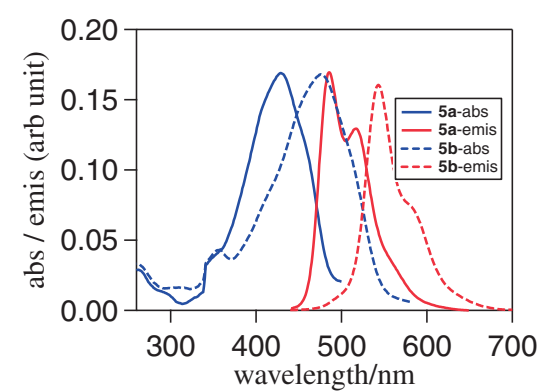

Figure 4. Comparison of absorption and emission spectra of allthiophene oligomers in $\mathrm{CHCl}_{3}$ at $\mathrm{rt}$.

property relationship. Multiple ZCP-containing thiophene oligomer precursors were prepared by this approach. Herein we have reported conversion of those intermediates to three kinds of derivatives and primarily studied UV-vis and emission spectra to realize their optical nature. Those oligothiophene derivatives have two active $\alpha-\mathrm{H}$ at thiophene ring in both ends which could be further manipulated to obtain desired efficiency.

This paper is in celebration of the 2010 Nobel Prize awarded to Professors Richard F. Heck, Akira Suzuki, and Ei-ichi Negishi.

\section{References and Notes}

1 I. F. Perepichka, D. F. Perepichka, H. Meng, F. Wudl, $A d v$. Mater. 2005, 17, 2281; A. Kraft, A. C. Grimsdale, A. B. Holmes, Angew. Chem., Int. Ed. 1998, 37, 402; G. A. Chamberlain, Sol. Cells 1983, 8, 47; H. E. Katz, Z. Bao, J. Phys. Chem. B 2000, 104, 671.

2 R. D. McCullough, Adv. Mater. 1998, 10, 93; S. Wu, L. Bu, L. Huang, X. Yu, Y. Han, Y. Geng, F. Wang, Polymer 2009, 50, 6245; A. Hucke, M. P. Cava, J. Org. Chem. 1998, 63, 7413; W. M. Albers, G. W. Canters, J. Reedijk, Tetrahedron 1995, 51, 3895; J. Roncali, Chem. Rev. 1997, 97, 173

3 a) T. Takahashi, F.-Y. Tsai, Y. Li, Chem. Lett. 1999, 1173. b) S. S. H. Mao, T. D. Tilley, Macromolecules 1997, 30, 5566.

4 For detailed synthetic procedure see Supporting Information. ${ }^{10}$

5 E.-i. Negishi, F. E. Cederbaum, T. Takahashi, Tetrahedron Lett. 1986, 27, 2829; E.-i. Negishi, T. Takahashi, Acc. Chem. Res. 1994, 27, 124.

6 T. Takahashi, F.-Y. Tsai, Y. Li, K. Nakajima, M. Kotora, J. Am. Chem. Soc. 1999, 121, 11093; T. Takahashi, F.-Y. Tsai, Y. Li, H. Wang, Y. Kondo, M. Yamanaka, K. Nakajima, M. Kotora, J. Am. Chem. Soc. 2002, 124, 5059; M. Hissler, P. W. Dyer, R. Réau, Coord. Chem. Rev. 2003, 244, 1; B. Jiang, T. D. Tilley, J. Am. Chem. Soc. 1999, 121, 9744; J. R. Nitschke, T. D. Tilley, J. Organomet. Chem. 2003, 666, 15; S. Yamaguchi, Y. Itami, K. Tamao, Organometallics 1998, 17, 4910; H.-C. Su, O. Fadhel, C.-J. Yang, T.-Y. Cho, C. Fave, M. Hissler, C.-C. Wu, R. Réau, J. Am. Chem. Soc. 2006, 128, 983, and references cited therein.

7 T. Takahashi, M. Kotora, Z. Xi, J. Chem. Soc., Chem. Commun. 1995, 361; T. Takahashi, Z. Xi, A. Yamazaki, Y. Liu, K. Nakajima, M. Kotora, J. Am. Chem. Soc. 1998, 120, 1672.

8 P. J. Fagan, W. A. Nugent, J. C. Calabrese, J. Am. Chem. Soc. 1994, 116, 1880.

9 Fluorescent quantum yield was calculated according to the procedure described: T. J. Russin, E. İ. Altinoğlu, J. H. Adair, P. C. Eklund, J. Phys.: Condens. Matter 2010, 22, 334217.

10 Supporting Information is available electronically on the CSJJournal Web site, http://www.csj.jp/journals/chem-lett/index. html. 